\title{
sciendo
}

Int. J. of Applied Mechanics and Engineering, 2019, vol.24, No.1, pp.179-198

DOI: 10.2478-ijame-2019-0012

\section{RHEOLOGY OF DRUGS FOR TOPICAL AND TRANSDERMAL DELIVERY}

\author{
A. WALICKA*, J. FALICKI \\ University of Zielona Góra, Faculty of Mechanical Engineering \\ ul. Szafrana 4, 65-516 Zielona Góra, POLAND \\ E-mails: A.Walicka@ijame.uz.zgora.pl \\ J.Falicki@ibem.uz.zgora.pl \\ B. IWANOWSKA-CHOMIAK \\ University Hospital of Zielona Góra, Oncology Department \\ ul. Zyty 26, 65-046 Zielona Góra, POLAND \\ E-mail: B.Chomiak@op.pl
}

\begin{abstract}
Skin drug delivery systems are a constant source of interest because of the benefits that they offer to overcome many drawbacks associated with other modes of drug delivery (i.e. oral, intravenous, etc.). Because of the impermeable nature of the skin, designing a suitable drug delivery vehicle that penetrates the skin barrier is challenging.

Skin drug delivery can be subdivided into topical and transdermal (Fig.1). In a topical administration the drug is intended to act at skin level, this is indicated for the treatment of skin diseases. The aim of transdermal administration is getting a systemic release and in this case the skin represents a barrier not a target. The availability of drugs or other active substances through the skin depends basically on two consecutive steps: the release of these drugs or substances from vehicle or carrier and their subsequent permeation through the skin. Hence, studies on the specific properties of vehicles or carriers, such as their rheological behaviours, are of great interest in the field of pharmaceutical products.

The objective of the present study is to systematically characterize a nonlinear rheological behaviour and flow properties of drugs and drug carriers into topical and transdermal administration. To this aim, one- and threedimensional rheological models are presented, which may be used to describe drug release through the skin and through the extracellular and interstitial matrix structures. Finally, the rheological measurements of some commercial creams and ointments were made.
\end{abstract}

Key words: drug delivery, human skin, drug rheology, rheological models.

\section{Introduction}

Skin is the human body's largest organ. It covers the entire body and separates the vital organs from the outside environment and serves as a protective barrier against physical, chemical and microbial attacks. Since the skin is the organ that is most exposed to the environment, the risk of damage of its integrity or the occurrence of a localized disease is very high [1-2].

The human skin is composed of three layers, consisting in: Stratum Corneum (SC), Epidermis and Dermis (Fig.1), the outer layer is the SC. The epidermis is put in contact with the dermis by so called dermoepidermis function (as a permeable membrane). The dermis is constituted by a connective tissue layer of mesenchymal origin. Finally, the inner layer is represented by the hypodermis, mainly constituted by connective tissue [3-11].

\footnotetext{
* To whom correspondence should be addressed
} 


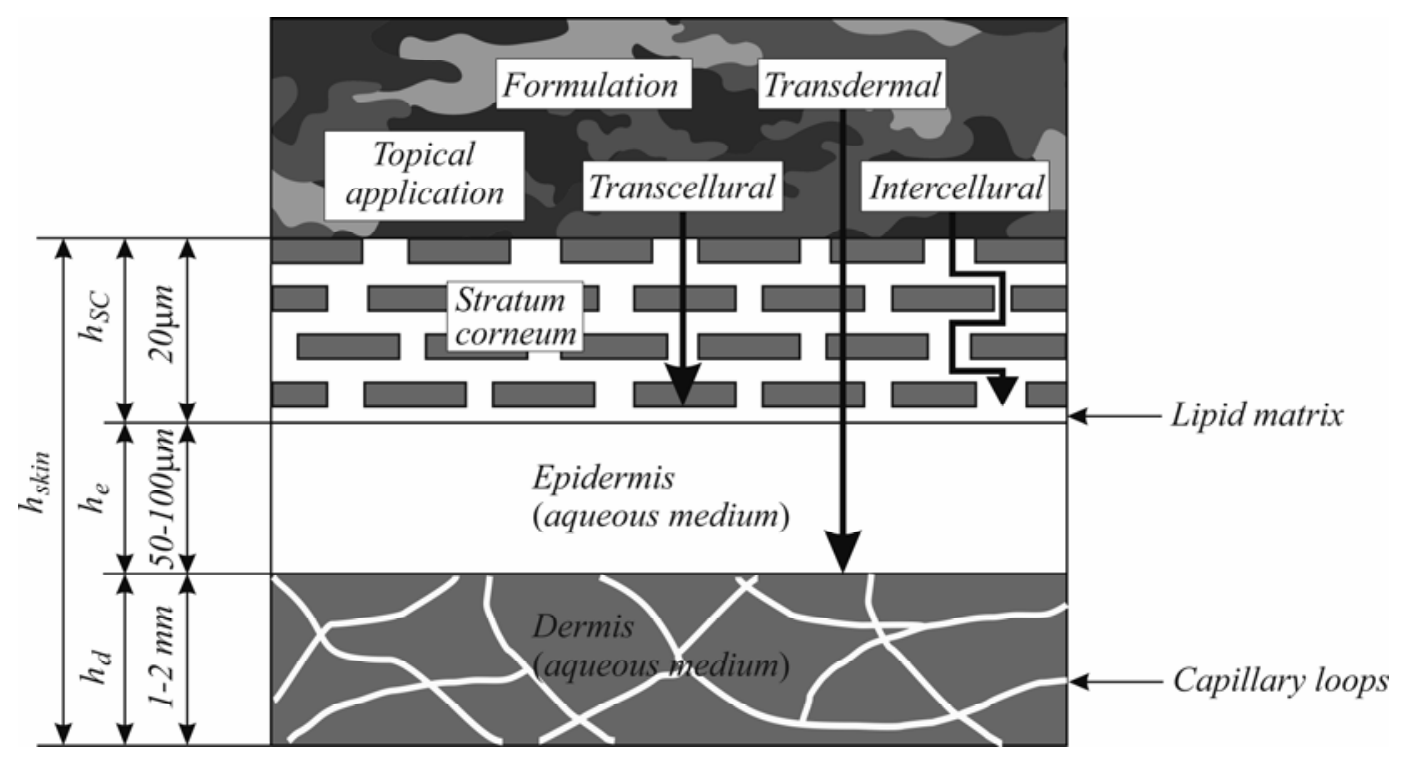

Fig.1. Schematic representation of the transport process involved in drug release from the formulation up to its uptake through the dermal capillaries.

Skin drug delivery can be subdivided into topical and transdermal. In a topical application the drug is intended to act at skin level. This delivery is an attractive route for local and systemic treatment. Topical drug administration may be localized anywhere in the body through ophthalmic, rectal, vaginal routes and skin. The delivery of drugs onto the skin is recognized as an effective means of therapy for local dermatologic diseases such as follicle-related disorders (e.g., acne and alopecias). It can penetrate deeper into skin and hence give better absorption $[12,13]$. Topical administrations have many advantages over the conventional dosage forms.

In general, they are deemed more effective and less toxic than conventional formulations due to the bilayer composition and structure. In the formulation of topical dosage forms, attempts are being made to utilize drug carriers that ensure adequate localization or penetration of the drug within or through the skin in order to enhance the local effects and minimize the systemic ones, or to ensure adequate percutaneous absorption.

Summarizing, it may be stated that the topical application of drugs offers potential advantages of delivering the drug directly to the site of action and acting for extended period of time. The drugs are intended for skin or for certain mucosal surfaces for their local action or percutaneous penetration of medicament or for their emollient or protective action.

The transdermal drug release is a variable administration route for powerful, not only low-molecular weight therapeutic agents, which has to be precise in the control of drug distribution. This release is specially recommended for many drugs that are difficult to be taken since they must be delivered slowly over a prolonged period to have a beneficial effect [7-10].

The transdermal drug administration has several interesting advantages over other systemic routes; the most interesting is the reduction of first-pass drug degradation as the liver is initially bypassed [4]. The transdermal drug delivery was mainly considered as diffusion transdermal delivery. Recently, methods of drug delivery taking into account the drug flow and the diffusion transport through the skin have been considered. The first application of these methods was described by Siegel [14] who approached insulin delivery by means of an implantable mechano-chemical pump converting changes in blood glucose concentration into a pressure pumping insulin out of the device. This pumping is possible after execution of micro-capillaries in the skin connecting the pump with the dermis, or - more generally - after an enhancement of the skin permeability. While chemical enhancers can be a successful tool for the improvement of small drug permeability through the skin, for big molecules other strategies have to be 
developed. Indeed, proteins and peptides transdermal administration requires the use of physical methods involving electrically based techniques (iontophoresis, electroporation, ultrasound, and photo-mechanical waves), structure-based techniques (microneedles, specially with internal jet-injection), and velocity-based techniques (jet-propulsion) [6-10, 15-20]. Each of these methods leads to the aforementioned execution of micro-capillaries in the skin.

The aim of this work is to present the rheological considerations on mathematical models of drugs and drug carriers _to be administered_through the skin. Motivated by the results of the recent papers on the drugs delivery through the skin, the principle idea of this work is to present new mathematical models describing the flow behaviour of novel drugs and experimental verification of some of these models. Finally, for all the models under consideration three dimensional forms of their descriptions are presented which may be used to model and analyse drug flows through the connective tissue [15-20].

\section{Structure of the skin delivery drugs}

Topical drug administration is recommended in skin diseases such as atopic dermatitis, psoriasis and ichthyosis; it is also advised in anti-inflammatory and in analgesic therapy [21,22].

In general, the topic and transdermal drug delivery are applied in dermatology, cosmetology, internal medicine and oncology [4].

Several pharmaceuticals such as creams, ointments, pastes and gels have been used for topical drug delivery, while suspensions, emulsions and also gels have been used for transdermal drug delivery [7].

Creams are two-phase systems containing oil and water, one of which is dispersed in the other in the form of microscope droplets (an emulsion). In order to achieve a homogeneous dispersion of the droplets, emulsifiers are added to the cream; some of these emulsifiers can cause an irritation of the skin [22]. In ointments the carrier fluid is more sticky than the water or oil and most frequently it is white vaseline or/and liquid paraffin [9].

Pastes are two-phase dilate systems with emulsifiers acting on droplets or solid particles; the carriers are: wax and its solutions [10]. oil [10].

Suspensions are two-phase systems of solid or fluid particles dispersed in carrier fluids such as water or

Emulsions are two-phase systems containing any kind of fluids dispersed in water; in emulsions the concentration of dispersed fluids is less than that in creams. We may differentiate microemulsions and nanoemulsions [7]. All these pharmaceuticals mentioned above may be - in particular cases -poly-phase dispersions and may be enriched into other drug agents.

Gels are defined as semisolid systems, which exhibit an external solvent phase. They are hydrophobic or hydrophilic in nature, and are immobilized within the spaces available of a threedimensional network structure [23-25]. Gels principally consist of a fluid solvent with the minority component forming a semisolid matrix; this matrix being a gelling agent such as carbomer or natural gum (e.g.: xantam or guar gum) is dispersed in purified water or in organic solvent to make a uniform dispersion. Gels, having more water than creams and ointments, permit greater dissolution of drugs and facilitate their migration through the vesicle and by their high water content they can hydrate the skin retaining a significant amount of transdermal water [26, 27]. One differentiates essentially two types of gels according to the nature of their liquid phase, namely: hydrogels containing water and organogels (oleogels) containing organic solvent.

Recently, some new trends and terminologies of gel systems in skin drug delivery have been noted. They are as follows: niosame and proniosame gels, emulgels, bigels, aerogels and xerogels [28].

Niosomes are liposomes consisting of a nonionic surfactant. They may be either unilamellar or multi-lamellar vesicles that are capable of carrying both hydrophilic and hydrophobic drugs.

Proniosomes are liquid crystalline compact niosomal hybrids, which may be converted into niosomes upon hydration. 
Emulgels are combinations of an emulsion and a gel. They may also include microemulgels and nano-emulgels, which reduce the diffusion barrier of the stratum corneum and show acceptable physical properties drug release and low skin irritations.

Bigels are topical formulations that are obtained by combining a hydrogel and an organogel, therefore they possess characteristics of both gels such as the cooling effect, enhancement of skin hydration, moisturizing, emollient effect, easy spreadability, and water-washability upon application to the skin.

Aerogels and xerogels are inorganic gels, since both are composed of silica but they undergo different drying procedures. If a wet silica gel is dried at normal pressure, it significantly shrinks and results in a dense material with a relatively small pores size known as a xerogel. In the case of a supercritical drying, shrinkage is avoided, the unusually porous structure is preserved, and the result is known as an aerogel.

\section{Rheological properties of the drugs and drug carriers}

Liquid drug carriers and pharmaceuticals are prepared from different substances having diverse physico-chemical and rheological properties. Most pharmaceutical and cosmetic processes such as new ingradient selections, formulation preparations, material packing and self storage are closely associated with complex flows of substances. The kind of applications and acceptance of pharmaceutical and cosmetics are also greatly dependent on the flow properties of the final products [29].

Therefore, knowledge of the rheological properties of pharmaceutical and cosmetic substances becomes an essential key to improve processing efficiency as well as to develop consumer acceptable final products.

Drug materials vary in consistency from solid to fluid state. An important category is to comprised of semi-solid products which are the most difficult substances to attempt a rheological characterization because they combine both solid-like and fluid-like properties within the same material. Widely used pharmaceutical and cosmetic products belonging to this category are ointments, creams, pastes and gels; all of which can be classified as semi-fluid materials.

Rheological measurements of these materials should be made for a number of reasons including [3036]:

- to understand the rheological nature of a system,

- to perform quality control of raw materials, final products and manufacturing processes (mixing, pumping, packing and filling),

- to study the effect parameters (formulation, storage time, temperature) on the quality of a final product,

- to assess a product with regard to actual usage such as spreading and adherence to the skin, removal from a tube or a jar.

\subsection{One dimensional models of rheologically complex drug carriers}

Taking into account the reasons mentioned above, the following one -dimensional model describing the rheological properties of pharmaceutical drug carriers [37] can be proposed:

- Shulman model

$$
\tau=\left[\tau_{0}^{1 / n}+(\mu \dot{\gamma})^{1 / m}\right]^{n}
$$

which is used to describe the flow properties of such pharmaceutical substances as creams, emulsions, ointments and pastes;

here: $\tau$ - is the shear stress, $\tau_{0}$ - is the yield shear stress, $\dot{\gamma}$ - is s the hear strain rate, $\mu$ - is the plastic viscosity, $m, n$ - are power exponents (material parameters). Note that by a reduction of coefficients in the Shulman model the formulae describing simpler models of the fluid can be obtained such as: 
- Newton: $m=n=1, \quad \tau_{0}=0$;

- Ostwald-de Waele: $n=1, \quad \tau_{0}=0$;

- Bingham: $m=n=1$;

- Herschel-Bulkley: $m \neq 0, \quad n=1$;

- Vočadlo: $m=1, \quad n \neq 0$;

- Casson: $m=n$ or $m=n=2$.

The Ostwald-de Waele model is frequently called the power-law model.

- Sisko model

$$
\tau=\left[\mu_{0}+\mu|\dot{\gamma}|^{n}\right] \dot{\gamma}
$$

which is applied to describe the flow properties of emulsions and suspensions here: $\mu_{0}$ - is the shear viscosity, $n$ - is power exponent. There are some models well describing the rheological drug properties which after suitable reduction of material parameters will be similar to the Sisko model.

These models are [37]:

Carreau-Yasuda

$$
\tau=\left\{\mu_{\infty}+\frac{\mu_{0}-\mu_{\infty}}{\left[1+(\kappa \dot{\gamma})^{n}\right]^{\frac{\alpha}{n}}}\right\} \dot{\gamma}
$$

Elsharkawy-Hamrock

$$
\tau=\frac{\mu_{0} \dot{\gamma}}{\left[1+(\kappa \dot{\gamma})^{n}\right]^{\frac{1}{n}}},
$$

Prandtl

$$
\tau=\mu_{0} \frac{\arcsin (\kappa \dot{\gamma})}{(\kappa \dot{\gamma})} \dot{\gamma}
$$

Eyring-Sutterby

$$
\tau=\mu_{0}\left[\frac{\operatorname{arsinh}(\kappa \dot{\gamma})}{\kappa \dot{\gamma}}\right]^{n} \dot{\gamma}
$$

Sutterby 


$$
\tau=\left\{\mu_{\infty}+\left(\mu_{0}-\mu_{\infty}\right)\left[\frac{\operatorname{arsinh}(\kappa \dot{\gamma})}{\kappa \dot{\gamma}}\right]^{n}\right\} \dot{\gamma},
$$

where $\mu_{0}, \mu_{\infty}$ - are limiting values of shear viscosity, $\kappa$ - is the material constant, $\alpha, n$ - are power exponents.

- DeHaven - Ellis model

$$
\tau=\frac{\mu_{0} \dot{\gamma}}{1+\kappa|\tau|^{n}}
$$

which may be used to describe the flow properties of micro- and nano-emulsions and suspensions. Similar models are given below [37]:

Meter

$$
\tau=\left[\mu_{\infty}+\frac{\mu_{0}-\mu_{\infty}}{1+(\kappa \tau)^{n}}\right] \dot{\gamma},
$$

Ree-Eyring

$$
\tau=\frac{\mu_{0} \dot{\gamma}}{\left[\frac{\sinh (\kappa \tau)}{(\kappa \tau)}\right]},
$$

Rabinowitsch

$$
\tau=\frac{\mu_{0} \dot{\gamma}}{1+\kappa \tau^{2}}
$$

Reiner-Philippoff

$$
\tau=\left[\mu_{\infty}+\frac{\mu_{0}-\mu_{\infty}}{1+(\kappa \tau)^{2}}\right] \dot{\gamma}
$$

Seely

$$
\tau=\left[\mu_{\infty}+\frac{\mu_{0}-\mu_{\infty}}{e^{(\kappa \tau)}}\right] \dot{\gamma}
$$

Note that all the above mentioned formulae - under some conditions - may be used for describing the flow properties of gels and emulgels. 
The gel being a mixed system of semisolid and semifluid substances often demonstrates viscoelastic behaviour. To describe the flow properties of such a system it is common to use the models of viscoelastic fluids.

The first of these models is usually not presented in the one dimensional notation but only to demonstrate its principle we will introduce this description [37]

$$
\tau=\mu \dot{\gamma}+\alpha_{I} \dot{\gamma}^{2}+\beta_{I}\left(\ddot{\gamma}+\dot{\gamma}^{2}\right)
$$

where $\ddot{\gamma}$ - is the velocity of the shear strain rate, $\alpha_{1}, \beta_{1}$ - are material coefficients. This model represents the so-called Rivlin-Ericksen viscoelastic fluid.

The second one is a Maxwell model [37]

$$
\tau=\mu \dot{\gamma}-\frac{\mu}{G} \dot{\tau}
$$

where $G$ is a Hookean elastic modulus.

\subsection{Three-dimensional models of rheologically complex drug carriers}

To find three-dimensional forms of the stress tensor $\boldsymbol{T}$, corresponding to the above given onedimensional forms of $\tau$, we may use the generalization whose basis is as follows [37]:

if

$$
\tau=f(\dot{\gamma}) \dot{\gamma}
$$

then

$$
(\boldsymbol{T})_{i j}=T_{i j}=-p \delta_{i j}+(\boldsymbol{\Lambda})_{i j}=-p \delta_{i j}+f(A)\left(\boldsymbol{A}_{1}\right)_{i j}
$$

where

$$
(\boldsymbol{\Lambda})_{i j}=\Lambda_{i j}=f(A)\left(\boldsymbol{A}_{l}\right)_{i j}
$$

here $p$ is the pressure, $\Lambda$ is the so-called extra-stress tensor, $\boldsymbol{A}_{l}$ is the first kinematic tensor of RivlinEricksen; here

$$
A=\left[\frac{1}{2} \operatorname{tr}\left(\boldsymbol{A}_{l}^{2}\right)\right]^{\frac{1}{2}}
$$

where $A$ is a square root from the second invariant of $\boldsymbol{A}_{l}$.

All equations of type

where

$$
\boldsymbol{T}=-p \mathbf{1}+\boldsymbol{\Lambda}, \quad \boldsymbol{\Lambda}=f(A) \boldsymbol{A}_{1}
$$

$$
(1)_{i j}=\delta_{i j}
$$

are called constitutive equations and generally they represent functional relations between the forces acting on the medium and the quantities describing the motion that may be realized, some of extra-stress tensors are presented in Tab.1. 
Table 1. Extra-stress tensors for pseudoplastic and viscoplastic fluids.

\begin{tabular}{|c|c|}
\hline Model of fluid & Form \\
\hline $\begin{array}{l}\text { (3.21) } \\
\text { power law or Ostwald-de Waele }\end{array}$ & $\boldsymbol{\Lambda}=\mu(A)^{n-1} \boldsymbol{A}_{1}$ \\
\hline $\begin{array}{l}(3.22) \\
\text { Sisko }\end{array}$ & $\boldsymbol{\Lambda}=\left[\mu_{0}+\mu(A)^{n}\right] \boldsymbol{A}_{1}$ \\
\hline $\begin{array}{c}(3.23) \\
\text { Carreau-Yasuda }\end{array}$ & $\boldsymbol{\Lambda}=\left\{\mu_{\infty}+\left(\mu_{0}-\mu_{\infty}\right)\left[1+(\kappa A)^{n}\right]^{-\alpha / n}\right\} \boldsymbol{A}_{1}$ \\
\hline $\begin{array}{l}(3.24) \\
\text { Prandtl }\end{array}$ & $\boldsymbol{\Lambda}=\left[\mu_{0} \frac{\arcsin (\kappa A)}{(\kappa A)}\right] \boldsymbol{A}_{l}$ \\
\hline $\begin{array}{l}(3.25) \\
\text { Eyring-Sutterby }\end{array}$ & $\boldsymbol{\Lambda}=\mu_{0}\left[\frac{\operatorname{arsinh}(\kappa A)}{\kappa A}\right]^{n} \boldsymbol{A}_{1}$ \\
\hline $\begin{array}{c}(3.26) \\
\text { Sutterby }\end{array}$ & $\boldsymbol{\Lambda}=\left\{\mu_{\infty}+\left(\mu_{0}-\mu_{\infty}\right)\left[\frac{\operatorname{arsinh}(\kappa A)}{\kappa A}\right]\right\}^{n} \boldsymbol{A}_{1}$ \\
\hline $\begin{array}{c}(3.27) \\
\text { DeHaven }\end{array}$ & $\Lambda=\frac{\mu_{0} A_{1}}{1+\kappa \Lambda^{n}}$ \\
\hline $\begin{array}{l}(3.28) \\
\text { Meter }\end{array}$ & $\boldsymbol{\Lambda}=\left[\mu_{\infty}+\frac{\mu_{0}-\mu_{\infty}}{1+\kappa \Lambda^{n}}\right] \boldsymbol{A}_{l}$ \\
\hline $\begin{array}{l}(3.29) \\
\text { Ree-Eyring }\end{array}$ & $\boldsymbol{\Lambda}=\left[\frac{\sinh (\kappa \Lambda)}{(\kappa \Lambda)}\right]^{-1} \mu_{0} A_{1}$ \\
\hline $\begin{array}{c}(3.30) \\
\text { Rabinowitsch }\end{array}$ & $\Lambda=\frac{\mu_{0} A_{1}}{1+\kappa \Lambda^{2}}$ \\
\hline $\begin{array}{l}\quad(3.31) \\
\text { Reiner-Philippoff }\end{array}$ & $\boldsymbol{\Lambda}=\left[\mu_{\infty}+\frac{\mu_{0}-\mu_{\infty}}{1+\kappa \Lambda^{2}}\right] \boldsymbol{A}_{l}$ \\
\hline $\begin{array}{l}(3.32) \\
\text { Seely }\end{array}$ & $\boldsymbol{\Lambda}=\left[\mu_{\infty}+\frac{\mu_{0}-\mu_{\infty}}{\exp (\kappa \Lambda)}\right] \boldsymbol{A}_{l}$ \\
\hline $\begin{array}{c}(3.33) \\
\text { Bingham }\end{array}$ & $\boldsymbol{\Lambda}=\left[\tau_{0}+\mu A\right] A^{-1} \boldsymbol{A}_{1}$ \\
\hline $\begin{array}{c}\text { (3.34) } \\
\text { Herschel-Bulkley }\end{array}$ & $\boldsymbol{\Lambda}=\left[\tau_{0}+(\mu A)^{l / m}\right] A^{-1} \boldsymbol{A}_{l}$ \\
\hline $\begin{array}{l}(3.35) \\
\text { Casson }\end{array}$ & $\boldsymbol{\Lambda}=\left[\tau_{0}^{l / n}+(\mu A)^{l / n}\right]^{n} A^{-1} \boldsymbol{A}_{l}$ \\
\hline $\begin{array}{c}(3.36) \\
\text { Shulman }\end{array}$ & $\boldsymbol{\Lambda}=\left[\tau_{0}^{l / n}+(\mu A)^{1 / m}\right]^{n} A^{-1} \boldsymbol{A}_{l}$ \\
\hline
\end{tabular}


Models of fluids from Eq.(3.21) to Eq.(3.26) represent the models of pseudoplastic fluids but the others represent the models of viscoplastic fluids:

here

$$
\Lambda=\left[\frac{1}{2} \operatorname{tr}\left(\Lambda^{2}\right)\right]^{\frac{1}{2}}
$$

is a square root from the second invariant of $\boldsymbol{\Lambda}$.

The constitutive relation for the second grade viscoelastic fluid of Rivlin-Ericksen is given as follows [37]

$$
\boldsymbol{T}=-p \boldsymbol{1}+\mu \boldsymbol{A}_{1}+\alpha \boldsymbol{A}_{1}^{2}+\beta \boldsymbol{A}_{2}
$$

where 1 is the unit tensor, $\mu, \alpha$ and $\beta$ are shear viscosity and material coefficient, respectively, and $\boldsymbol{A}_{1}, \boldsymbol{A}_{2}$ are the first two Rivlin-Ericksen tensors defined by

$$
\begin{aligned}
& \boldsymbol{A}_{l}=\boldsymbol{L}+\boldsymbol{L}^{T}, \quad \boldsymbol{A}_{l}^{2}=\boldsymbol{A}_{l} \cdot \boldsymbol{A}_{l}=\left(\boldsymbol{L}+\boldsymbol{L}^{T}\right)^{2}, \quad \boldsymbol{L}=\operatorname{grad} \mathbf{v}, \\
& \boldsymbol{A}_{2}=\dot{\boldsymbol{A}}_{l}+\boldsymbol{A}_{l} \boldsymbol{L}+\boldsymbol{L}^{T} \boldsymbol{A}_{l}, \quad \dot{\boldsymbol{A}}_{l}=\frac{d \boldsymbol{A}_{l}}{d t}=\left[\frac{\partial}{\partial t}+(\mathbf{v} \cdot \nabla)\right] \boldsymbol{A}_{l}
\end{aligned}
$$

symbol ${ }^{T}$ denotes the transpose of the tensor matrix.

The constitutive relation for the Maxwell fluid is given as follows [37]

$$
\boldsymbol{\Lambda}+\lambda \frac{d \mathbf{\Lambda}}{d t}=\mu \boldsymbol{A}_{1}
$$

where $\lambda$ is a new material coefficient having a dimension of $\mu / G$ or dimension of time $t$. Another similar viscoelastic fluid is a Jeffreys fluid for which the constitutive equation has the form

$$
\boldsymbol{\Lambda}+\lambda_{1} \frac{d \boldsymbol{\Lambda}}{d t}=\mu\left(\boldsymbol{A}_{1}+\lambda_{2} \frac{d \boldsymbol{A}_{1}}{d t}\right)
$$

where $\lambda_{1}, \lambda_{2}$ are material coefficient having a dimension of time $t$.

\section{Constitutive equations for generalized viscoelastic fluids}

Many fluids of medical interest appear to exhibit yield behaviour, where flow occurs only when the imposed stress exceeds a critical yield stress. To describe the most general rheological behaviour of such a viscoplastic fluid the non-linear model of Shulman (3.36) may be used; it is suitable to present this model in the following form

$$
\boldsymbol{T}=-p \mathbf{1}+M \boldsymbol{A}_{1}, \quad M=\left[\frac{1}{\tau_{0}^{n}}+(\mu A)^{\frac{1}{m}}\right]^{n}
$$

where $M$ is called a viscosity function. 
To obtain a model that does exhibit both viscoplastic and viscoelastic behaviour Walicki and Walicka proposed the following two constitutive equations for generalized second grade fluids [37]

$$
\boldsymbol{T}=-p \mathbf{I}+M \boldsymbol{A}_{1}+\alpha_{1} \boldsymbol{A}_{1}^{2}+\beta_{1} \boldsymbol{A}_{2}
$$

or

$$
\boldsymbol{T}=-p \boldsymbol{1}+M\left(\boldsymbol{A}_{1}+\alpha_{2} \boldsymbol{A}_{1}^{2}+\beta_{2} \boldsymbol{A}_{2}\right) .
$$

Considering some recent experimental and theoretical results Walicka [37] proposed certain generalization of models given by Eqs (3.42) and (3.43) assuming that $M=M_{i}$, where $M_{i}$ represents the viscosity functions given in Tab.2.

Table 2. Viscosity function $M_{i}$ for some non-Newtonian fluids.

\begin{tabular}{|c|c|}
\hline Model & Form of $M_{i}$ \\
\hline $\begin{array}{c}(4.4) \\
\text { Newton }\end{array}$ & $M_{i}=\mu$ \\
\hline $\begin{array}{c}(4.5) \\
\text { Ostwald-de Waele }\end{array}$ & $M_{i}=\mu(A)^{n-1}$ \\
\hline $\begin{array}{c}(4.6) \\
\text { Sisko }\end{array}$ & $M_{i}=\mu_{0}+\mu(A)^{n}$ \\
\hline $\begin{array}{c}(4.7) \\
\text { Carreau-Yasuda }\end{array}$ & $M_{i}=\mu_{\infty}+\frac{\mu_{0}-\mu_{\infty}}{\left[1+(\kappa A)^{n}\right] \alpha / n}$ \\
\hline $\begin{array}{c}(4.8) \\
\text { Prandtl }\end{array}$ & $M_{i}=\mu_{0} \frac{\arcsin (\kappa A)}{(\kappa A)}$ \\
\hline$(4.9)$ & $M_{i}=\mu_{0}\left[\frac{\arcsin (\kappa A)}{(\kappa A)}\right]^{n}$ \\
\hline Eyring-Sutterby & $M_{i}=\mu_{\infty}+\left(\mu_{0}-\mu_{\infty}\right)\left[\frac{\operatorname{arcsinh}(\kappa A)}{(\kappa A)}\right]^{n}$ \\
\hline $\begin{array}{c}(4.10) \\
\text { Sutterby }\end{array}$ & \\
\hline
\end{tabular}

Note that these generalized second grade fluids may be used to describe the flows of drugs and drug carriers being emulsions, suspensions, and even lipid based lyotropic liquid crystals [38, 39]. These crystals can be mainly classified into lamellar phase $\left(L_{\alpha}\right)$ cubic phase $\left(Q_{2}\right.$ or $\left.V_{2}\right)$ and hexagonal phase $\left(H_{2}\right)$ according to their different internal structures. Among them $V_{2}$ and $H_{2}$ are the most important and recently they have received much attention due to their highly ordered internal structure, which offers the great potential as a slow release matrix for active pharmaceutical ingredients with various size and polarities [40-44]. Cubic and hexagonal liquid crystals are often formed by the addition of certain amphiphilic lipids in an aqueous environment (e.g., they can be prepared by simply blending lipid and aqueous phase by vortex). When both 
these liquid crystals are dispersed into nanoparticles with excess water with addition of certain stabilizers, they form stable colloidal dispersions which are termed cubosomes and hexosomes, respectively.

Analysing the latest reports on the rheological behaviour of lyotropic liquid crystals presented in the papers [40-44], it seems that novel descriptions relating to viscoelastic fluids of Maxwell and Jeffreys type being drug carriers may be provided. These descriptions consist in changing the simple material derivatives of type $\dot{\boldsymbol{T}}=\frac{d \boldsymbol{T}}{d t}$, used in Eqs (3.40) and (3.41), to more complex derivatives, such as: $\nabla, \Delta, \frac{\mathcal{D}}{\mathcal{D} t}$ and even $\square$ (see Appendix); then the constitutive equations for both these fluids, in current notations, will be [37]

- Maxwell type

$$
\Lambda+\lambda \stackrel{\square}{\Lambda}=\mu \stackrel{\square}{\boldsymbol{A}_{1}}
$$

- Jeffreys type

$$
\Lambda+\lambda_{1} \stackrel{\stackrel{口}{\Lambda}}{=} \mu\left(A_{1}+\lambda_{2} \stackrel{口}{A}_{l}\right)
$$

where symbol $\square$ denotes so called Oldroyd general derivative (see Eq.(A.11) in Appendix).

Let us still consider the specific models of viscoelastic fluids being drug carriers called fractional models. In order to describe the viscoelastic properties of fluids in various medical fields more exactly, equations with besides the constitutive even complex derivatives, we would like to introduce, fractional time derivatives [37].

Then the constitutive equation of a viscoelastic fluid for the fractional Maxwell model has the form

$$
\left(1+\lambda^{\alpha} \frac{d^{\alpha}}{\partial t^{\alpha}}\right) \Lambda=\mu \boldsymbol{A}_{1} .
$$

In the above expression $\alpha(0 \leq \alpha \leq 1)$ is the fractional viscoelastic constant, and the Riemann-Liouville derivative operator $\partial^{\alpha} / \partial t^{\alpha}$ is expressed by the following equation

$$
\frac{d^{\alpha}}{d t^{\alpha}} f(t)=\frac{1}{\Gamma(1-\alpha)} \frac{d}{d t} \int_{0}^{t} \frac{f(z)}{(t-z)^{\alpha}} d z
$$

in which $\Gamma(\bullet)$ is a Gamma function. It is noted that for $\alpha=1$, Eq.(4.14) reduces to a Maxwell model given by Eq.(3.40).

Similarly, the fractional model for a Jeffreys fluid has the form

$$
\left(1+\lambda_{1}^{\alpha} \frac{d^{\alpha}}{d t^{\alpha}}\right) \Lambda=\mu\left(1+\lambda_{2}^{\beta} \frac{d^{\beta}}{d t^{\beta}}\right) \boldsymbol{A}_{l}
$$

where $\alpha$ and $\beta$ are the fractional parameters such that $0 \leq \alpha \leq \beta \leq 1$ and $\alpha \leq \beta$; the derivative operator according to Eq.(A.1) is expressed as follows 


$$
\frac{d^{\alpha} \boldsymbol{T}}{d t^{\alpha}}=\left[\frac{\partial^{\alpha}}{\partial t^{\alpha}}+(\mathbf{v} \cdot \nabla)\right] \boldsymbol{T} .
$$

Replacing the derivative $\frac{d^{\alpha}}{\partial t^{\alpha}}$ by $\frac{\mathcal{D}^{\alpha}}{\mathcal{D} t^{\alpha}}$ (see Eq.(A.4) in Appendix), we will obtain the so called generalized fractional models for which

$$
\frac{\mathcal{D}^{\alpha} \boldsymbol{T}}{\mathcal{D} t^{\alpha}}=\frac{d^{\alpha} \boldsymbol{T}}{d t^{\alpha}}+\boldsymbol{T} \boldsymbol{W}-\boldsymbol{W T}
$$

\section{Rheometric measurements}

All rheological measurements serve to determine the material coefficients such as: $\mu, \mu_{0}, \mu_{\infty}, \kappa, m, n, \tau_{0}$, for pseudoplastic and viscoplastic fluids and $G, \alpha, \beta, \lambda, \lambda_{1}, \lambda_{2}$ for other fluids of Maxwell or Jeffreys type. Different methods which are used to determine and measure these material parameters are described in [45-48]. Generally, instruments named rheometers are used for these measurements. Instruments which are limited in their use only for the measurements of the viscous flow behaviour of fluids are called viscometers. The action of viscometers consists in an analysis of shear induced flow in fluids which can occur in four model cases [45]:

- flow between two parallel discs, when one disc moves and the other is stationary;

- flow in the annular gap between two concentric cylinders, when one is assumed to be stationary while the other rotates;

- flow through pipes, tubes or capillaries;

- flow between two parallel discs or between cone and disc sensor systems when one is stationary and the other rotates.

\subsection{Method and materials}

All rheological analyses were performed for some typical commercial creams and ointments with a controlled-stress rheometer VT550 (Haake, Karlsruhe-Germany) and RheoWin 4.50 software, with cone - and - disc sensor system of $28 \mathrm{~mm}$ diameter with a gap of $50 \mu \mathrm{m}$ (height of removed tip in truncated cone) and cone angle $\alpha=1^{\circ}$. The measurements will be limited only to find so called flow curves being a graphical form of the expression

$$
\tau=f(\dot{\gamma})
$$

The shear rate $\dot{\gamma}_{c}$ is equal to [45]

$$
\begin{aligned}
& \dot{\gamma}_{c}=\frac{1}{\tan \alpha} \cdot \Omega=M \cdot \Omega\left[s^{-1}\right], \\
& M=\frac{1}{\tan \alpha} \approx \frac{1}{\alpha}, \quad \Omega=\frac{2 \pi n}{60}
\end{aligned}
$$


where:

$\Omega$ is the angular velocity $[\mathrm{rad} / \mathrm{s}]$,

$n$ is the rotor speed $\left[\mathrm{min}^{-1}\right]$,

$\alpha$ is the cone angle $[\mathrm{rad}]$,

$M=57.300[(1 / \mathrm{s}) /(\mathrm{rad} / \mathrm{s})]$ is the shear rate factor; it is a constant for the cone - and - disc sensor system used in our experiment.

The shear stress is equal to [45]

$$
\tau_{c}=\left(\frac{3}{2 \pi R_{c}^{3}}\right) M_{d}=A \cdot M_{d}
$$

where:

$\tau_{c}$ is the shear stress on the cone $[P a]$,

$R_{c}$ is the outer radius of the cone $[m]$,

$M_{d}$ is the torque to be measured on the rotor axis $[\mathrm{Nm}]$,

$A=174000.000[\mathrm{~Pa} / \mathrm{Nm}]$ is the shear stress factor; it is a constant for the sensor system.

To find the flow curves the following commercial creams and ointments were used:

1. Diclofenac $10 \mathrm{mg}$ (known also as Voltaren 1\% ) - topical gel,

2. Hydrocortisonum $0.5 \%$ - cream,

3. Voltaren Max 2.3\% (diclofenac $23 \mathrm{mg}$ ) - topical gel,

4. Nitrocard $20 \mathrm{mg}$ (known as nitroglycerine) - topical gel,

5. MaxiBiotic $5 m g$ (known as neomycine) - ointment.

The rheological measurements were performed on both the up and down curves with shear rates over the range $0.01-1000 \mathrm{l} / \mathrm{sec}$ and back, each stage lasting $60 \mathrm{~s}$ with a stay lasting $30 \mathrm{~s}$. This range was selected on the basis of the strength of resistance to the applied stress. The flow curves were fitted to the following models:

- Ostwald deWaele

$$
\tau=\mu \dot{\gamma}^{n}
$$

- Casson

$$
\tau=\left[\tau_{0}^{l / n}+(\mu \dot{\gamma})^{l / n}\right]^{n} \quad n=2
$$

- Herschel-Bulkley 


$$
\tau=\tau_{0}+\mu \dot{\gamma}^{n}
$$

All tests were carried out on samples of about $0.1 \mathrm{~cm}^{3}$ at two temperatures $20^{\circ} \pm 0.1^{\circ} \mathrm{C}$ (storage temperature) and $36.6^{\circ} \pm 0.1^{\circ} \mathrm{C}$ (health use temperature).

The results are presented in Figs 2-4 and Tabs 3-5; note that in these figures and tables Voltaren Max is describes as Voltaren.

It follows from the presented results that with a very high probability (with the coefficient of regression $r^{2} \geq 0.98$ ) one may select theoretical flow curves of different models to describe the real flow curves.
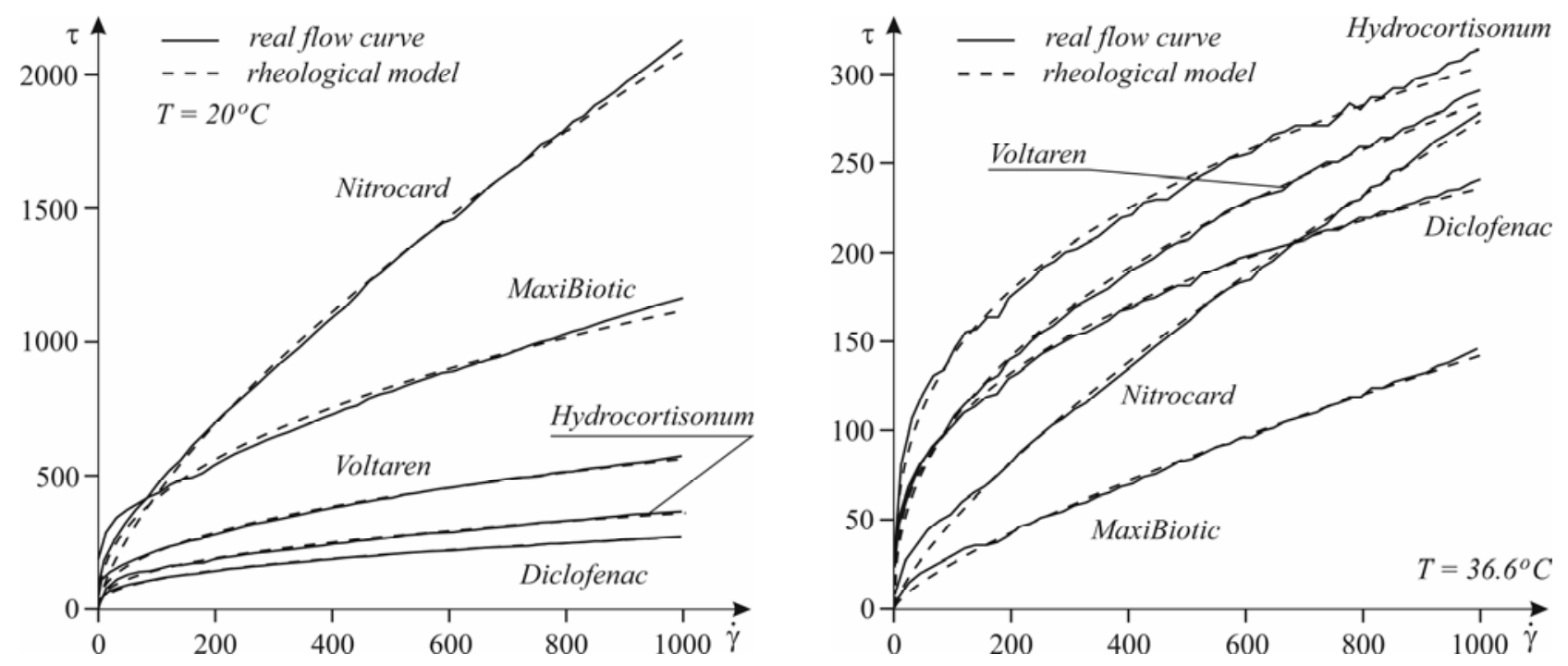

Fig.2. Real flow curves and flow curves corresponding to the Ostwald deWaele model.

Table 3. Rheological parameters of flow curves corresponding to the Ostwald deWaele (power-law) model.

\begin{tabular}{|c|c|c|c|c|}
\hline \multicolumn{5}{|c|}{ Ostwald deWaele fluid $\tau=\mu \dot{\gamma}^{n}$} \\
\hline paran & \multicolumn{2}{|c|}{$T=20^{\circ} \mathrm{C}$} & \multicolumn{2}{|c|}{$T=36.6^{\circ} \mathrm{C}$} \\
\hline substances & {$\left[\begin{array}{c}\mu \\
{\left[P a \cdot s^{n}\right.}\end{array}\right]$} & $\begin{array}{l}n \\
-\end{array}$ & $\begin{array}{c}\mu \\
{\left[P a \cdot s^{n}\right.}\end{array}$ & $\begin{array}{l}n \\
-\end{array}$ \\
\hline Diclofenac & 17.93 & 0.3930 & 19.71 & 0.3594 \\
\hline Hydrocortisonum & 23.77 & 0.3931 & 30.98 & 0.3306 \\
\hline MaxiBiotic & 57.47 & 0.4298 & 0.794 & 0.7503 \\
\hline Nitrocard & 18.42 & 0.6848 & 1.561 & 0.7481 \\
\hline Voltaren & 31.88 & 0.4153 & 14.37 & 0.4317 \\
\hline
\end{tabular}



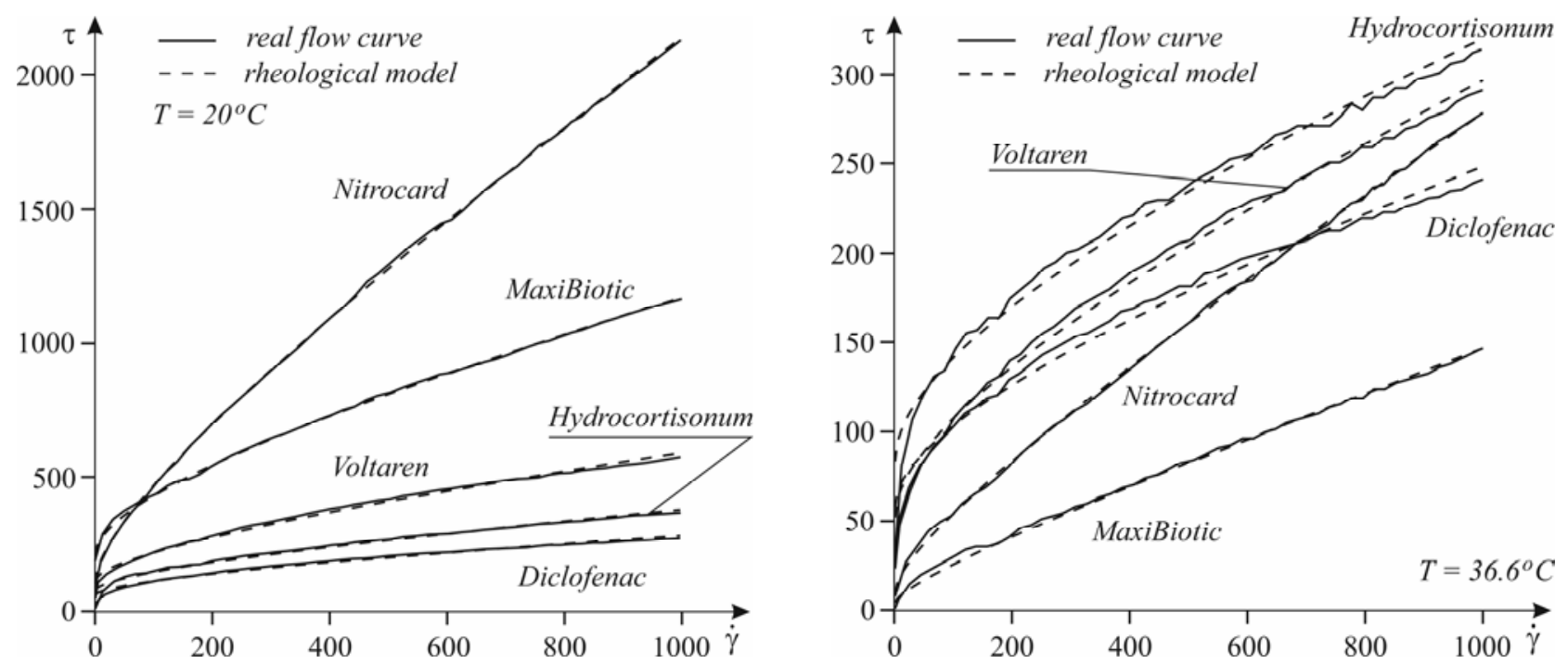

Fig.3. Real flow curves and flow curves corresponding to the Casson model.

Table 4. Rheological parameters of flow curves corresponding to the Casson model.

\begin{tabular}{|c|c|c|c|c|}
\hline \multicolumn{3}{|c|}{ Casson fluid $\tau=\left[\tau_{0}^{1 / n}+(\mu \dot{\gamma})^{1 / n}\right]^{n} n=2$} \\
\hline parameters & \multicolumn{2}{|c|}{$T=20^{\circ} \mathrm{C}$} & \multicolumn{2}{|c|}{$T=36.6^{\circ} \mathrm{C}$} \\
\cline { 2 - 5 } & $\begin{array}{c}\tau_{0} \\
{[P a]}\end{array}$ & $\begin{array}{c}\mu \\
{[P a \cdot s]}\end{array}$ & $\begin{array}{c}\tau_{0} \\
{[P a]}\end{array}$ & $\begin{array}{c}\mu \\
{[P a \cdot s]}\end{array}$ \\
\hline substances & 60.69 & 0.0814 & 57.00 & 0.0674 \\
\hline Diclofenac & 75.89 & 0.1148 & 82.81 & 0.0774 \\
\hline Hydrocortisonum & 210.60 & 0.3869 & 3.00 & 0.1073 \\
\hline MaxiBiotic & 106.10 & 1.2900 & 8.95 & 0.1877 \\
\hline Nitrocard & 108.80 & 0.1913 & 51.42 & 0.1011 \\
\hline Voltaren & & & & \\
\hline
\end{tabular}

Examining the descending flow curves of the rheograms shown in Figs 2-4 it can be seen that all drugs present a non-Newtonian character at both temperatures. Analyzing these curves it can be observed that the tested pharmaceuticals may be numbered both among the pseudoplastic substances and the viscoplastic substances. As it is noted in Tabs 4 and 5 , the yield stress $\tau_{0}$ decreases with the increasing temperature; Hydrocortisonum is here an exception. The yield stress increases with the increasing diclofenac concentration (compare Diclofenac $10 \mathrm{mg}$ with Voltaren Max containing $23 \mathrm{mg}$ of diclofenac).

Plastic viscosity $\mu$ and flow index $n$ follow the same trends without an exception as the yield stress with respect to the temperature changes.

\section{Conclusions}

In this paper, we have focused on an analytical description of rheological properties of the drugs for topical and transdermal delivery. For this purpose we considered rheological models of drugs having a different consistency: suspension, gel, cream etc, etc. 

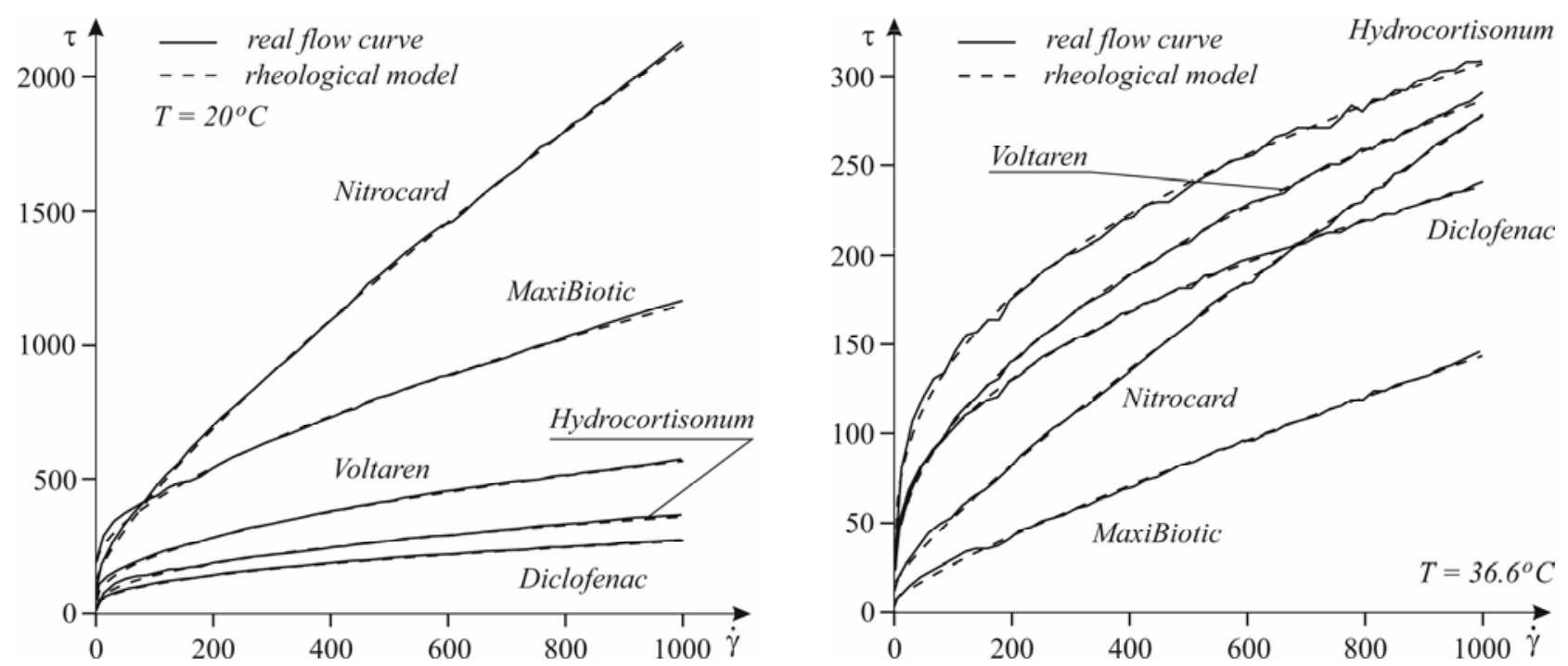

Fig.4. Real flow curves and flow curves corresponding to the Herschel-Bulkley model.

Table 5. Rheological parameters of flow curves corresponding to the Herschel-Bulkley model.

\begin{tabular}{|c|c|c|c|c|c|c|}
\hline \multicolumn{7}{|c|}{ Herschel-Bulkley fluid $\tau=\tau_{0}+\mu \dot{\gamma}^{n}$} \\
\hline \multirow{3}{*}{ parameters } & \multicolumn{3}{|c|}{$T=20^{\circ} \mathrm{C}$} & \multicolumn{3}{|c|}{$T=36.6^{\circ} \mathrm{C}$} \\
\cline { 2 - 8 } & $\begin{array}{c}\tau_{0} \\
{[P a]}\end{array}$ & $\mu$ & $n$ & $\tau_{0}$ & $\mu$ & $n$ \\
{$\left[P a \cdot s^{n}\right]$} & - & {$[P a]$} & {$\left[P a \cdot s^{n}\right]$} & - \\
\hline substances & 26.11 & 10.42 & 0.458 & 25.14 & 10.320 & 0.4386 \\
\hline Diclofenac & 32.75 & 15.96 & 0.439 & 33.40 & 16.840 & 0.4037 \\
\hline Hydrocortisonum & 195.70 & 13.00 & 0.622 & 5.719 & 0.472 & 0.8213 \\
\hline MaxiBiotic & 129.80 & 8.795 & 0.785 & 16.13 & 0.720 & 0.8535 \\
\hline Vitrocard & 49.04 & 17.55 & 0.490 & 23.36 & 8.033 & 0.5051 \\
\hline
\end{tabular}

First, the simplest models of fluids such as pseudoplastic and viscoplastic fluids represented by the Shulman models, then more complicated models of viscoplastic fluids such as Maxwell and Jeffreys models were considered, and finally, fractional models of viscoelastic fluids were analysed. It seems that the latter may be used to describe the rheological behaviour of such drug carriers as lyotropic liquid crystals.

Knowledge of rheological behaviour of pharmaceuticals designed for topical and transdermal application is very important. The knowledge can be used at technological level e.g. by an insertion of active or auxiliary substances and at therapeutical level when the rheological parameters can be correlated with composition of drugs and therefore with their consistency, bioavailability and also their stability.

Rheological testing performed on the same commercial pharmaceuticals showed that the all formulations had a non-Newtonian pseudoplastic or viscoplastic behaviour at both considered temperatures.

\section{Appendix}

Formulae (3.39) $)_{5}$ and (3.39) $)_{4}$ define two types of derivatives. Besides these derivatives one uses other types of derivatives in rheology. Let us consider a few examples of the definitions of time derivatives used in the theory of constitutive equations. They are as follows [37]:

- material derivative (see:Eq. (3.39) $\left.)_{4}\right)$ 


$$
\dot{\boldsymbol{T}}=\frac{d \boldsymbol{T}}{d t}=\left[\frac{\partial}{\partial t}+(\mathbf{v} \cdot \nabla)\right] \boldsymbol{T}
$$

- lower Oldroyd convective derivative

$$
\stackrel{\Delta}{\boldsymbol{T}}=\dot{\boldsymbol{T}}+\boldsymbol{T} \boldsymbol{L}+\boldsymbol{L}^{T} \boldsymbol{T},
$$

- upper Oldroyd convected derivative

$$
\stackrel{\nabla}{\boldsymbol{T}}=\dot{\boldsymbol{T}}-\boldsymbol{T} \boldsymbol{L}^{T}-\boldsymbol{L} \boldsymbol{T}
$$

- co-deformational or co-rotational Zaremba-Jaumann

$$
\stackrel{\circ}{\boldsymbol{T}}=\frac{\mathcal{D} \boldsymbol{T}}{\mathcal{D} t}=\dot{\boldsymbol{T}}+\boldsymbol{T} \boldsymbol{W}-\boldsymbol{W} \boldsymbol{T}
$$

where

$$
\boldsymbol{W}=\frac{1}{2}\left(\boldsymbol{L}-\boldsymbol{L}^{T}\right)
$$

is called the spin tensor;

note that this derivative coincides with a half of the sum of the Oldroyd derivatives

$$
\stackrel{\circ}{\boldsymbol{T}}=\frac{1}{2} \stackrel{\Delta}{\boldsymbol{T}}+\frac{1}{2} \stackrel{\nabla}{\boldsymbol{T}}
$$

- generalized Zaremba-Jaumann derivative

$$
\frac{\mathcal{D}_{a} \boldsymbol{T}}{\mathcal{D} t}=\stackrel{o}{\boldsymbol{T}}+a(\boldsymbol{T D}+\boldsymbol{D T}),
$$

where

$$
\boldsymbol{D}=\frac{1}{2}\left(\boldsymbol{L}-\boldsymbol{L}^{T}\right)
$$

- Gordon-Schowalter general derivative

$$
\stackrel{\square}{\boldsymbol{T}}=\frac{a}{2} \stackrel{\Delta}{\boldsymbol{T}}+\left(1-\frac{a}{2}\right) \stackrel{\nabla}{\boldsymbol{T}}, \quad 0 \leq a \leq 2
$$

or

$$
\stackrel{\square}{\boldsymbol{T}}=\frac{1-\varsigma^{\Delta}}{2} \stackrel{\Delta}{\boldsymbol{T}}+\frac{1+\varsigma}{2} \stackrel{\nabla}{\boldsymbol{T}}, \quad-1 \leq \varsigma \leq 1
$$

also being a specific generalization of the Zaremba-Jauman derivative,

- Oldroyd general derivative 


$$
\stackrel{\square}{\boldsymbol{T}}=\boldsymbol{J}_{a b c} \boldsymbol{T}=\stackrel{o}{\boldsymbol{T}}+a(\boldsymbol{T} \boldsymbol{D}+\boldsymbol{D} \boldsymbol{T})+b(\boldsymbol{T}: \boldsymbol{D}) \boldsymbol{1}+c \boldsymbol{D} \operatorname{tr} \boldsymbol{T},
$$

where $a, b$ and $c$ are arbitrary chosen constants; in particular, we have

$$
\boldsymbol{J}_{100} \boldsymbol{T}=\stackrel{\Delta}{\boldsymbol{T}} \quad \text { and } \quad \boldsymbol{J}_{-100} \boldsymbol{T}=\stackrel{\nabla}{\boldsymbol{T}}
$$

Here the symbol (:) denotes the scalar (or double) product of two tensors (in our case $\boldsymbol{T}$ and $\boldsymbol{D}$ ).

\section{Nomenclature}

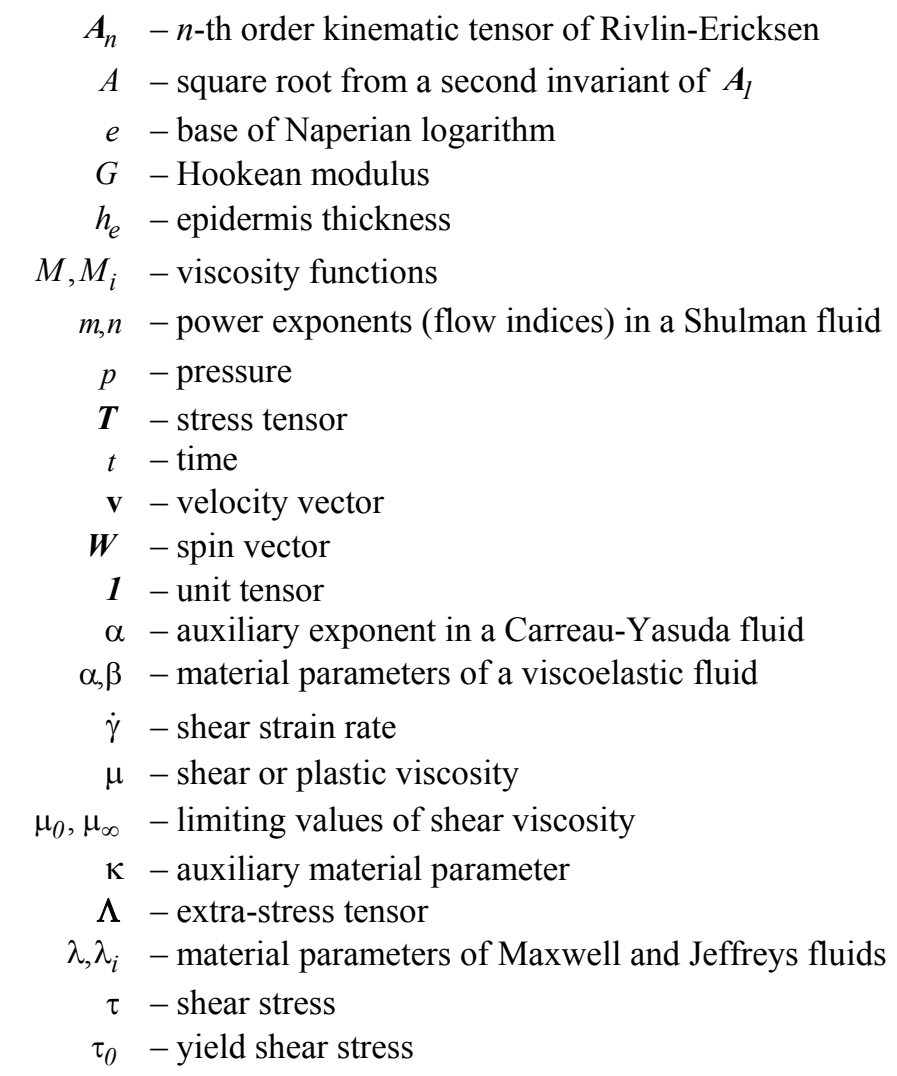

\section{References}

[1] Lukas L. (1988): The epidermal permeability barrier. - Anat. Embryol, No.178, pp.1-13.

[2] Peter M.E. (1988): Structure and function of stratum corneum permeability barrier. - Drug Develop. Res., vol.13, No.1, pp.97-105.

[3] Kalia Y.N. and Guy R.H. (2001): Modeling transdermal drug release. - Adv. Drug Delivery Rev., vol.48, pp.159-172.

[4] Carreras N., Alonso C., Marti M. and Lis M.J. (2015): Mass transport model through the skin by microencapsulation system. - J. Microencapsulation, vol.32, No.4, pp.358-363.

[5] Milington P.F. and Wilkinson R. (1983): Skin. - Cambridge University Press.

[6] Cross S.E. and Roberts M.S. (2004): Physical enhancement of transdermal drug application: Is delivery technology keeping up with pharmaceutical development? - Curr. Drug Deliv. vol.1. No.1, pp.81-92. 
[7] Grassi M. (2008): Membranes in drug delivery. - In: Handbook of Membrane Separations: Chemical, Pharmaceutical, Food and Biotechnological Applications (Pabby A.K., Rizvi S.S.H. and Sastre A.M., Eds), pp.427-464.

[8] Prausnitz M.R. and Langer R. (2008): Transdermal drug delivery. - Nat. Biotechnol., vol.26, No.11, pp.1261-1268.

[9] Anisimov Y.G. and Roberts M.S. (2014): Mathematical models for topical and transdermal drug products. - In: Topical Drug Bioavailability, Bioequivalence and Penetration (Shah V.P., Maibach H.I. and Jenner J., Eds), Second ed., Springer, New York, pp.249-298.

[10] Benson H.A.E. (2005): Transdermal drug delivery: Penetration enhancement techniques. - Curr. Drug. Deliv., vol.2, No.1, pp.23-33.

[11] Walicka A. and Iwanowska-Chomiak B. (2018): Drug diffusion transport through the human skin. - Int. J. of App. Mech. Eng., vol.23, No.4, pp.977-988.

[12] Mikari B.V. and Machadik K.R. (2010): Formulation and evaluation of typical liposomal gel for fluconozole. Ind. J. Pharm Sci., vol.44, No.4, pp.324-325.

[13] Scheuplein R.J. (2013): A personal view of skin permeation. - Skin Pharm. Physiol., vol.26, No.1, pp.199-212.

[14] Siegel R.A. (1990): PH-sensitive gels: Swelling equilibria, kinetics and applications for drug delivery. - In: Pulsed and Self-Regulated Drug Delivery (Kost J. Ed.), CRC Press, New York, pp.129-157.

[15] Benson H.A.E. (2005): Transdermal drug delivery: Penetration enhancement techniques. - Curr. Drug. Deliv., vol.2, No.1, pp.23-33.

[17] Arora A., Prausnitz M.R. and Mitragori S. (2007): Micro-scale devices for transdermal drug delivery. - Int. J. Pharm., No.364, pp.227-236.

[18] Cal K. (2009): Across skin barrier; known methods, new performances. - In: Frontiers in Drug Design and Discovery, vol.4 (Caldwell G.W., Ur-Rahman A., Yan Z., Choudhary M.J., Eds), Bentham Science Publisher, New York, pp.162-188.

[19] Cal K. and Stefanowska J. (2010): Methods for skin permeation enhancement of drug substances. - Technology of drug form, vol.66, No.7, pp.514-520.

[20] Walicka A. and Iwanowska-Chomiak B. (2018): Fractal model of the transdermal drug delivery. - Int. J. App. Mech. Eng., vol.23, No.4, pp.989-1004.

[21] Proksch E., Fölster-Holst R. and Jensen J.M. (2006): Skin barrier function, epidermal proliferation and differentiation in eczema. - J. Dermatol. Sci., vol.43, No.1, pp.159-169.

[22] Bouwstra J.A. and Ponec M. (2006): The skin barrier in healthy and diseased state. - Biochoim. biophys. Acta, No.1758, pp.2080-2095.

[23] Abdallah D.J and Weiss R.G. (2000): Organogels and low molecular mass organic gelators. - Adv. Mat., vol.12, No.12, pp.1237-47.

[24] Goyal S., Sharma P., Ramchandani U., Shrivastava S.K. and Dubey P.K. (2011): Novel anti-inflammatory topical gels. - Int. J. Pharm. Biol. Arch., vol.2, No.4, pp.1087-1094.

[25] Vintiloiu A. and Leroux J.C.(2008): Organogels and their use in drug delivery - a review. - J. Control Release, No.125, pp.179-192.

[26] Shapiro Y.E. (2011): Structure and dynamics of hydrogels and organogels: an NMR spectroscopy approach. Prog. Polym. Sci., vol.36, No.4, pp.1184-1254.

[27] Allan S.H. (2012): Hydrogels for biomedical applications. - Adv. Drug Deliv. Rev., vol.64, No.1, pp.18-23.

[28] Anisimov Y.G. and Roberts M.S. (2014): Mathematical models for topical and transdermal drug products. - In: Topical Drug Bioavailability, Bioequivalence, and Penetration (Shah V.P., Maibach H.I., and Jenner J. Eds), Second ed., New York, pp.249-298.

[29] Park E.-K. and Song K.-W. (2010): Rheological evaluation of petroleum jelly as a base material in ointment and cream formulations: steady shear flow behavior. - Arch. Pharm. Res., vol.33, No.1, pp.141-150. 
[30] Carvalho F.C., Calixto G., Hatakeyama I.N., Luz G.M., Gremião M.P.D. and Chorilli M. (2013): Rheological, mechanical, and bioadhesive behavior of hydrogels to optimize skin delivery systems. - Drug Develop. Ind. Pharmacy, vol.39, No.11, pp.1750-1757.

[31] Oliveira M.B., Haddad do Prado A., Bernegossi J., Sato C.S., Brunetti I.L., Scarpa M.V., Leonardi G.R., Friberg S.E. and Chorilli M. (2014): Topical application of retinyl palmitate-loaded nanotechnology-based drug delivery systems for the treatment of skin aging. - BioMed Res. Int., vol.2014, art ID 632570, 7pp.

[32] Isaac V.L.B., Chiari-Andréo B.G., Marto J.M., Moraes J.D.D., Leone B.A., Corrêa M.A and Ribeiro H.M. (2015): Rheology as a tool to predict the release of alpha-lipoic acid from emulsions used for the prevention of skin aging. - BioMed Res. Int., vol.2015, art ID 818656, 8pp.

[33] Ferreira P.G., Souza, R.S., Costa V.G. Ferreira V.F., Cruz R.C. and Futuro D.O. (2017): Development and characterization of emulgels for treatment of chronic wounds. - Asian J. Pharm. Tech. Inn., vol.5, No.25, pp.40-48.

[34] Ortan A., Dinu-Parvu C., Ghica M.V., Popescu L.M. and Ionita L. (2011): Rheological study of a liposomal hydrogel based on carbopol. - Rom. Biotech. Letters, vol.16, No.1, pp. 47-54.

[35] Ghica M.V., Hîrjău M., Lupuleasa D. and Dinu-Pîrvu C.-E. (2016): Flow and thixotropic parameters for rheological characterization of hydrogels. - Molecules, vol.21, 786; doi:10.3390/molecules21060786.

[36] Lucero M.J., Ferris C., Sánchez-Gutiérrez C.A., Jiménez-Castellanos M.R. and de-Paz M.-V. (2016): Novel aqueous chitosan-based dispersions as efficient drug delivery systems for topical use. Rheological, textural and release studies. - Carbohydrate Polymers, vol.151, pp.692-699.

[37] Walicka A. (2017): Rheology of Fluids in Mechanical Engineering. - Zielona Góra: University Press.

[38] Guo C., Wang J., Cao F., Lee R. and Zhai G. (2010): Lyotropic liquid crystal systems in drug delivery. - Drug Discovery Today, vol.15, No.23/24, pp.1032-1040.

[39] Chen Y., Ma P. and Gui S. (2014): Cubic and hexagonal liquid crystals as drug delivery systems. - BioMed Res. Int. vol.2014, art. ID 815981, 12 pp.

[40] Nazaruk E., Miszta P., Filipek S., Górecka E., Landau E.M. and Bilewicz R. (2015): Lyotropic cubic phases for drug delivery: diffusion and sustained release from the mesophase evaluated by electrochemical methods. Langmuir, vol.46, No.31, pp.12753-12761.

[41] Kulkarni C.V., Vishwapathi V.K., Quarshie A., Moinuddin Z., Page J., Kendrekar P. and Mashele S.S. (2017): Self-assembled lipid cubic phase and cubosomes for the delivery of aspirin as a model drug. - Langmuir, vol.33, No.38, pp.9907-9915.

[42] Astolfi P., Giorgini E., Gambini V., Rossi B., Vaccari L., Vita F., Francescangeli O., Marchini C. and Pisani M. (2017): Lyotropic liquid-crystalline nanosystems as drug delivery agents for 5-fluorouracil: structure and cytotoxicity. - Langmuir, vol.33, No.43, pp.12369-12378.

[43] Chountoulesi M., Pippa N., Pispas S., Chrysina E.D., Forys A., Trzebicka B. and Demetzos C. (2018): Cubic lyotropic liquid crystals as drug delivery carriers: physicochemical and morphological studies. - Int. J. Pharm. No.550, pp.57-70.

[44] Huang Y. and Gui S. (2018): Factors affecting the structure of lyotropic liquid crystals and the correlation between structure and drug diffusion. - Roy. Soc. Chem. Adv., vol.8, pp.6978-6987.

[45] Schramm G. (1994): A Practical Approach to Rheology and Rheometry. - Karlsruhe: Haake.

[46] Walters K. (1975): Rheometry. - London: Chapman\&Hall.

[47] Ferguson J. and Kembłowski Z. (1991): Applied Fluid Rheology. - London: Elsevier Appl. Sci.

[48] Whorlow R.W. (1992): Rheological Techniques. - New York: Ellis Horwood.

Received: June 18, 2018

Revised: September 6, 2018 